\title{
Enfermería: la Némesis de la ciencia y filosofía del cuidado
}

\author{
Rey Arturo Salcedo Álvarez,* Sara Huerta González,** Sebastián Bustamante Edquén****
}

\section{RESUMEN}

En la cultura griega, para proteger el equilibrio universal la diosa Némesis castigaba la desmesura. A partir de esta metáfora se realizó un ejercicio crítico desde la concepción teórica, epistemológica y práctica de la enfermería, centrándose en explorar el concepto de cuidado en las dimensiones de interacción, participación y significado. A partir de este análisis, se concluyó que existe un distanciamiento entre los aspectos teóricos y filosóficos con la práctica cotidiana de la profesión.

Palabras clave: Enfermería, cuidado, filosofía, epistemología.

\section{Nursing: the Nemesis of science and philosophy of care}

\begin{abstract}
In Greek culture, to protect the universal balance the goddess Nemesis punished excess. From this metaphor a critical exercise was made of the theoretical, epistemological and practical conception of nursing, focusing on exploring the concept of care in the dimensions of interaction, participation and meaning. From this analysis, it was concluded that there is a critical distance between theoretical and philosophical aspects with the daily practice of the profession.
\end{abstract}

Key words: Nursing, caring, philosophy, epistemology.

\section{INTRODUCCIÓN}

$\mathrm{L}$ a palabra Némesis no forma parte del diccionario de la Real Academia Española; sin embargo, para la mitología griega, Némesis era la diosa de la justicia retributiva, la venganza y la fortuna. Su función era castigar la desmesura y proteger el equilibrio universal.

Trasladando esta metáfora al quehacer científico de enfermería, el papel de los investigadores es generar conocimiento para diseñar, contribuir y profundizar en la abstracción y praxis del desarrollo de tecnologías aplicadas al cuidado de la salud de las personas con un enfoque humanista, solidario y respetuoso. Sin embargo, se observa que gran parte del cúmulo de conocimientos logrados, más que dirigido a procurar acciones de promoción y educación para que las personas sanas permanezcan sanas, está orientado a la curación de la enfermedad. Tal parece que el personal de salud es personal de contención de la enfermedad; es por esto que se plantean los siguientes supuestos y un axioma:

\footnotetext{
* Licenciado en Enfermería y Obstetricia, Maestro en Salud Pública, Profesor de Carrera Asociado "C”, Doctorado en Ciencias de la Enfermería Universidad Nacional de Trujillo, Perú. Secretario de Planeación y Evaluación. ENEO-UNAM.

** Licenciada en Enfermería, Maestra en Ciencias de la Enfermería, Doctorada en Ciencias de la Enfermería Universidad Nacional de Trujillo, Perú. Docente por Asignatura de la Universidad Veracruzana, Área Materno Infantil Facultad Enfermería, Universidad Veracruzana. *** Enfermero, Doctor en Enfermería y Especialista en Administración Hospitalaria y Gestión de la Salud. Profesor Principal de la Facultad de Enfermería y EPG de la Universidad Nacional de Trujillo, Perú.
}

Correspondencia: Rey Arturo Salcedo Álvarez. Camino Viejo a Xochimilco s/n y Viaducto, Tlalpan, Col. San Lorenzo Huipulco, 14370, México, D.F., E-mail: rasalced@hotmail.com 
Supuesto 1: La ciencia, por ser una construcción social, está al servicio del desarrollo humano de manera incluyente, democrática, justa retributiva y equitativa.

Supuesto 2: A partir del cuidado de la vida y de la salud, la ciencia en enfermería coadyuva al desarrollo humano.

Axioma 1: El vínculo que asocia el discurso con la práctica de enfermería está en construcción.

Valga esta metáfora, los dos supuestos y el axioma como punto de partida para exponer las siguientes reflexiones.

\section{DISCUSIÓN}

A mediados de abril pasado, el diario francés "Le Monde" publicó un artículo censurado de Albert Camus (1913-1960) que en 1939 abogaba por el ejercicio libre del periodismo en tiempos de guerra; en éste afirmaba que "una verdad expresada en tono dogmático es censurada nueve de cada diez veces", mientras que "la misma verdad, dicha irónicamente, sólo lo es cinco de cada diez". ${ }^{1}$

Enfermería ha recurrido pocas veces a filósofos existencialistas; se autodenomina "La ciencia del cuidado" y parte del supuesto de que es una profesión humanista, cuando en realidad casi todo el trabajo cotidiano y la mayor parte del conocimiento desarrollado se sustenta aún en el paradigma de la enfermedad desde un enfoque clínico, fragmentado, curativo, medicalizado y técnico-mecanizado, prácticamente carente de acciones que promuevan la salud; de ahí que los fundamentos y preceptos filosóficos y la producción científica no están, propiamente, encauzados a proveer salud sino, en el mejor de los casos, a restablecer la salud de las personas enfermas.

En México, enfermería, como disciplina científica formadora de recursos humanos profesionales con diseños curriculares propios, tendrá escasamente cinco décadas; es probable que ésta sea una de las causas por las que aún prevalece cierta dificultad para integrar de manera sincrónica, en la práctica cotidiana, los conceptos de sujeto de estudio, objeto de trabajo, ciencia, filosofía y praxis. Sin embargo, en los últimos años el cuidado de enfermería se ha convertido en eje central de la reflexión sobre el futuro de la disciplina, misma que obligadamente involucra al análisis de las tendencias filosóficas que cuestionan y defienden el cuidado como un acto que no es propio ni exclusivo de la profesión de enfermería, planteando la necesidad de aclarar el significado de este concepto para la disciplina desde los más profundos aspectos filosóficos y epistemológicos.

Johannes Hessen (1889-1971) afirmó que la filosofía es un intento del espíritu humano para llegar a una concepción del universo mediante la autorreflexión sobre sus funciones valorativas teóricas y prácticas; la filosofía, entendida como una reflexión sobre la conducta teórica, le llamaríamos "Ciencia" y, por tanto, la "Filosofía" es teoría del conocimiento científico o teoría de la ciencia. ${ }^{2}$ Aplicando estos preceptos a la disciplina, esto equivaldría a profundizar en el fundamento humanista sobre el cuidado.

Así mismo, en relación con la ciencia Mario Bunge describe que "mientras los animales inferiores sólo están en el mundo, el hombre trata de entenderlo; y sobre la base de su inteligencia imperfecta pero perfectible del mundo, el hombre intenta enseñorearse de él para hacerlo más confortable. En este proceso, construye un mundo artificial: ese creciente cuerpo de ideas llamado ciencia". ${ }^{3}$

Ahora bien, retomando estos enfoques en enfermería, se debe asumir que el conocimiento obtenido de manera racional mediante la investigación científica ha contribuido al avance en la concepción de la salud y el cuidado de la vida de las personas. Así mismo, Edgar Morín considera que "La comprensión" incluye dos tipos: a) la intelectual, relacionada a lo que puede ser explicado por la inteligencia; y b) la basada en la experiencia humana, que implica la relación de aprehensión sujeto-sujeto y el desarrollo de un proceso de empatía, identificación y proyección. Estos dos tipos de comprensión interaccionan con ciertas estructuras arraigadas en los seres humanos que se deben superar para vencer la incomprensión a través de la educación. Por lo tanto, el problema de la incomprensión se resolvería educando para la comprensión humana. ${ }^{4}$

En este sentido, la profesión de enfermería debe contribuir a profundizar en la discusión filosófica y científica, para tender así un puente entre el cuidado profesional, concebido como una profunda expresión de humanismo, y la dignidad de las personas que cuidamos.

Por consiguiente, para tratar de comprender el trabajo cotidiano de enfermería el "sujeto" es la persona que cuida y el "objeto" la persona cuidada; la cierto es que en el trabajo de enfermería, aun el producto de la investigación se orienta hacia procedimientos esenciales en el ámbito clínico hospitalario, centrados en personas enfermas con miras a la recuperación de "su salud".

Leonardo Boff (1938- ), en su obra El cuidado esencial hace alusión al "modo-de-ser-cuidado". Según su visión, éste se compone y se armoniza con el "modo-de-ser-en-el-mundo" como una forma de existir y coexistir, de estar presente y de la relación arco-existencia y con-vivencia en donde la relación es sujeto-sujeto, en una relación de convivencia, interacción y comunión. Para Boff, cuidar implica intimidad, sentimientos, acogida, respeto, consuelo, sosiego y reposo; es decir, el centro ya no está ocupado por el Logos (razón) sino por el phatos (sentimiento); de esta manera se 
permite al ser humano vivir la experiencia fundamental del valor, y a partir de ello, surgen las dimensiones de alteridad, sacralidad, reciprocidad y complementariedad. ${ }^{5}$

Asumiendo lo planteado por Boff, y desde nuestro punto de vista, el cuidado puede, con mucha propiedad, adaptarse en la práctica de la enfermera, dándole una nueva perspectiva de ética. En este sentido, el gran desafío para la enfermera es encontrar la medida justa entre el trabajo relacionado con la materialidad y el cuidado vinculado con la espiritualidad. Si bien la salud se entiende como un proceso dinámico, eje de las acciones de cuidado, a la fecha la práctica de enfermería se ha enfocado de manera casi exclusiva en el cuidado de personas enfermas, es decir, hemos sido copartícipes del desarrollo de tecnologías aplicadas. Derivado de esta situación, la investigación y el trabajo de enfermería se han centrado en atender la enfermedad más que en el cuidado de la salud, como si el objeto de enfermería, más que la salud, fuera el cuidado de la enfermedad. Por lo tanto, la salud y la enfermedad constituyen el mejor ejemplo de la uni-dualidad humana: al llegar las enfermedades, éstas se quedan y nunca más se van; sin embargo, una enfermedad humanamente vivida contribuye a descubrir y mejorar la salud sufragando la felicidad en otras dimensiones de la vida.

Por ello es importante la incursión de enfermería en la investigación científica. Esto es relativamente reciente: lo muestran las primeras investigaciones disciplinares realizadas en Estados Unidos hace 50 años, enfocadas a procesos clínicos. En la última década, se ha alcanzado el mayor crecimiento en la generación de conocimiento en enfermería, con un enfoque comprensivo, hermenéutico y fenomenológico.

Suzanne Kérouac refiere que la enfermera, como gestora del cuidado de pacientes, familias, comunidades y poblaciones, se responsabiliza de planificar, organizar, dirigir y controlar los recursos financieros, humanos y materiales con la intención de atender, de la mejor manera y eficacia posibles, los objetivos de la organización; por consiguiente, la enfermera deberá de tener siempre presente al enfermo en su contexto familiar, social y cultural para saber qué prefiere el paciente y dejarle escoger un tratamiento. ${ }^{6}$

En los últimos 100 años, los avances en la enseñanza, profesionalización e investigación de la disciplina han sido evidentes; sin embargo, el objeto de estudio de la enfermería (léase el cuidado de la vida de las personas, familias, comunidades sanas y enfermas) se ha centrado en el aspecto individual, clínico y resolutivo de la enfermedad, principalmente en las personas ya enfermas, sin descubrir su dimensión, presencia de la enfermedad y por tanto la coexistencia de la salud junto a la enfermedad, y al no visualizar una enfermedad se deja prácticamente de lado la atención a las personas sanas.?

\section{CONSIDERACIONES FINALES}

Se pretende lograr que los niveles de salud sean verdaderamente un coadyuvante para lograr un mejor equilibrio en el desarrollo humano más incluyente, democrático, justo y equitativo; tal vez sea el momento de replantear los conceptos de enfermería, salud y persona, así como el cuidado con ética y amor.

La interacción sujeto-objeto en el ámbito de la enfermería es la conjunción del ser para hacer y el hacer para ser; en el primero estaríamos hablando de generar el ámbito teórico para sustentar nuestra práctica y en el segundo generar nuevos conocimientos para sustentar no sólo una mayor identidad profesional, sino para imbuir mayor conciencia sobre el papel que enfermería debería tener como personal de salud y elemento precursor en la redefinición de salud y cuidado con un enfoque social en donde los principios de autonomía, respeto a la cultura y forma de organización sean parte esencial de la práctica cotidiana y no sólo mero discurso.

La perspectiva actual tiene una marcada influencia en el enfoque centrado en la enfermedad, situación que ha limitado el quehacer profesional. Es necesario reubicar la profesión de enfermería no sólo como coadyuvante en la recuperación de la salud, sino como un conjunto de ciencia y filosofía que trabaje para que las personas sanas permanezcan sanas, es decir, que su objeto de estudio sean también las personas, familias y comunidades sanas y desarrollar de esta manera mayor investigación para demostrar cómo, en el ámbito de lo sano, la enfermería puede generar conocimientos que favorezcan su desarrollo científico y humano.

El objetivo de la enfermería se dirige al estudio del cuidado de la vida de los seres humanos con un enfoque histórico eco-organizacional; significa la instrumentación del cuidado de todas las vivencias, necesidades y respuestas de las personas ante la búsqueda de su realización. Este objeto de cuidado es a la vez sujeto con cuerpo y espíritu, único e irrepetible, cuidado por otro cuerpo y espíritu que se autorrealiza profesional y personalmente al ejercer esta práctica. Así, la investigación sobre el cuidado generará conocimiento para atender a las personas sin separarlas en "enfermas" o "sanas", buscando un equilibrio de la disciplina que sea incluyente, democrático, justo y equitativo, y aplicar de tal forma un conocimiento basado en la ciencia y la filosofía al desarrollo del bienestar humano.

En vez de continuar caminando en "líneas paralelas", donde una de éstas corresponde a la ciencia y su filosofía y la otra a la praxis enfocada a la recuperación de la salud a través de la curación, debemos esforzarnos por lograr que este avance se realice en líneas convergentes. Recordemos que en la cultura griega la diosa Némesis castigaba la desmesura para proteger 
el equilibrio universal; por consiguiente, la enfermería tiene la necesidad de entender el cuidado, en el sentido de equilibrar prevención-recuperación, ciencia-filosofía, para evitar la venganza de Némesis ante la negligencia por no aceptar la felicidad en la enfermedad y la infelicidad en la salud.

\section{REFERENCIAS}

1. Le Monde 17 de march de 2012. Un appelinédit de Camus. Disponible en: http://www.lemonde.fr. Recuperado el 19 de marzo de 2012.

2. Hessen J. Teoría del conocimiento. Disponible en: Recuperado el 8 de marzo de 2012. http://www.libroos.es/libros-de-filosofia/ epistemologia/3583-hessen-johannes-teoria-del-conocimiento-pdf.html
3. Bunge M. La ciencia, su método y su filosofía. Recuperado el 8 de marzo de 2012. Disponible en www.philosophia.cl/Escuela de Filosofía Universidad ARCIS.

4. Morín E. Los siete saberes necesarios para la educación del futuro. 1999 , UNESCO. Disponible en: http://www.unmsm.edu.pe/occaa/articulos/ saberes7.pdf . Recuperado el 12 de marzo de 2012.

5. Boff L. El cuidado esencial. Colección estructuras y procesos. Madrid: Ed. Trotta; 2002.

6. Kerouac $\mathrm{S}$ et al. Propuesta para la gestión de los cuidados. En: El Pensamiento Enfermero. Barcelona, España: Ed. Masson; 1996. pp. 120-147.

7. Dos Santos I. Sociopoética: un puente para cuidar-investigar en enfermería. Index de enfermería. 2005; 14 (50) Disponible en: http://scielo. isciii.es/scielo.php?script=sci-arttex\&pid=S1132-12962005000200007. Recuperado el 25 de septiembre de 2012. 